Research Article

\title{
Experimental Study on Lateral Permeability Evolution of Sandstone under Seepage-Damage Coupling
}

\author{
Dongdong Pang, ${ }^{1,2,3}$ Feisheng Feng $\left(\mathbb{D},{ }^{1,3}\right.$ Guanghui Jiang $\left(\mathbb{D},{ }^{4}\right.$ Dongjing Xu $\left(\mathbb{D},{ }^{5}\right.$ \\ and Xiaohan Wang $\mathbb{1 D}^{2}$ \\ ${ }^{1}$ State Key Laboratory of Mining Response and Disaster Prevention and Control in Deep Coal Mine, \\ Anhui University of Science and Technology, Huainan 232001, China \\ ${ }^{2}$ School of Mining and Safety Engineering, Anhui University of Science and Technology, Huainan 232001, China \\ ${ }^{3}$ Key Laboratory of Coal Mine Safety and Efficiently Caving of Ministry of Education, \\ Anhui University of Science and Technology, Huainan, Anhui 232001, China \\ ${ }^{4}$ School of Civil Engineering, Ludong University, Yantai 264025, China \\ ${ }^{5}$ Shandong Prov Key Lab Deposit Mineralizat \& Sedim, Shandong University of Science and Technology, Qingdao 266590, \\ Shandong, China \\ Correspondence should be addressed to Feisheng Feng; fsfeng21@qq.com
}

Received 1 June 2020; Revised 24 September 2020; Accepted 15 October 2020; Published 29 October 2020

Academic Editor: Hailing Kong

Copyright (C) 2020 Dongdong Pang et al. This is an open access article distributed under the Creative Commons Attribution License, which permits unrestricted use, distribution, and reproduction in any medium, provided the original work is properly cited.

\begin{abstract}
To analyze the internal pore evolution law of aquifer rock in a coal mining front under the coupling effect of stress and seepage and the influence on the water inrush performance of the working face, research on the working face was conducted to improve the RTR-1000 high-temperature and high-pressure rock triaxial mechanical testing system, using hollow cylindrical and complete sandstone samples and by considering the stress change law in the actual mining process as the reference loading path. At the initial stage of loading, the permeability of sandstone demonstrates a rapid downward trend within a small range of stress change, with a decline rate of $50 \%$. At lower permeabilities, the fluctuation is small; the plastic and failure stages are transient, and the relationship curve between the horizontal permeability and the axial confining pressure of sandstone is divided into compaction, multiple fluctuations, surrender, and failure. In several stages, the sandstone lateral permeability experiment under the coupling effect of stress and seepage demonstrates that the permeability of the aquifer in the coal mining front is significantly reduced after the original rock stress is disturbed by mining, suggesting that the water inrush calculation of the traditional water-flowing fractured zone and caving zone aquifer rock permeability experiment is inaccurate. Further research can deepen the stress and seepage coupling evolution process under the action of working face water inrush.
\end{abstract}

\section{Introduction}

Underwater coal mining inevitably involves problems such as water gushing on the working face, water inrush into the tunnel, and groundwater seepage. The interaction between the coal strata and groundwater influences the coal seam and groundwater, and there exists a coupling problem between seepage and stress. The coupling of seepage and stress is manifested mainly when seepage occurs; the water pressure caused by seepage acts on the rock and changes its condition.
Changes in the external influences of rock stress change the internal crack seepage features, seepage, and stress coupling mainly through the seepage characteristics of the fracture [1-6]. Snow determined the stress under the action of the permeability coefficient expression through a set of parallel fracture experiments [7]. Oda used statistical methods to express the tensor of the crack and obtain the relationship between stress and seepage [8-10]. Barton et al. proposed a relationship between the permeability coefficient and the effective stress by studying the mechanical properties of 
fractures [11]. Reichenberger et al. proposed a finite volume method for vertex centers that can be used for the complete coupling of two-phase flow in porous media fractures [12]. Hoteit and Firoozabadi proposed an effective numerical model of incompressible two-phase flow in cracked media [13]. Helmig et al. studied the model coupling of multiphase flow in porous media [14]. Peratta and Popov developed a new numerical method for three-dimensional (3D) modeling of flows and transient solute transport in cracked porous media, thereby providing accurate and effective treatment of 3D complex geometric shapes and inhomogeneity [15]. Zhao et al. proposed a stress and seepage coupling research method based on digital image technology under the same conditions [16]. The digital image was obtained by segmentation, and the spatial distribution information was extracted. Linear interpolation is a 3D digital analysis model that was established to determine the stress of the spatial structure and seepage spatial division of the cataclastic rock mass characteristics. Wang and Zhang proposed a numerical simulation method for fracture propagation and closure under seepage stress coupling that was modeled using the extended finite element method based on the research background of fractures and voids [17]. Gui et al. reported that the primary cause of karst water inrush was the coupling effect of mining stress and bottom seepage [18]. Yi, Xiao, Tan, Ferfera, Chen, and Sheng-Qi Yang et al. conducted rock damage experiments on rocks of different lithologies and concluded that the permeability of rocks changes significantly after damage [19-24]. Shan and Lai, Sheikh and Pak, Guo et al., and Zhang et al. conducted related research on stressing osmotic coupling using numerical simulation, establishing numerical models of rock stress-strain-permeability coupling, and preliminary studies on the mechanism of stress-seepage coupling [25-28]. Ding and $\mathrm{Xu}$, Shi and Durucan, Weeks, and Wang and Yakushev. conducted permeability studies in different directions under the condition of convective solid coupling [29-32]. The results indicated that permeability in different directions varies significantly under the same experimental conditions. The aforementioned research has laid the theoretical foundation for investigation in this study of the coupling of underground stress and permeability under the condition of damage caused by coal mining. However, there has been little experimental research on the change rule of horizontal permeability under mining pressure, particularly for the pseudotriaxial rock mechanics test system. The stress application can be conducted in two ways. In the first method, vertical in situ stress under confining pressure and horizontal in situ stress under axial pressure and confining pressure are applied to test the change law of axial permeability of rock with increasing confining pressure. The horizontal in situ stress in two directions is significantly different from the actual horizontal in situ stress, and the applied magnitude of the vertical in situ stress can only be smaller than that of the horizontal in situ stress, which is significantly different from the actual conditions. In the second method, the vertical in situ stress is applied under axial pressure, and the horizontal in situ stress is applied under confining pressure, which better simulates the actual stress. The conventional permeability measurement can only measure the axial permeability; thus, the permeability measurement method must be improved to obtain the lateral permeability measurement of the conventional pseudotriaxial apparatus.

\section{Materials and Methodology}

2.1. Experimental Design. The China University of Mining and Technology (Beijing) RTR-1000 high-temperature and high-pressure rock triaxial mechanical testing system can implement program design through rock experiments under different stress conditions of stress path loading, which creates conditions for simulating the stress change environment of in situ experiments. The permeability test does not include a test of transverse permeability; thus, the permeability test of the test system was modified to enable the measurement of the lateral permeability of the rock specimen. The rock specimen was sandstone; its buried depth was $270 \mathrm{~m}$, bulk density was $20.5 \mathrm{kN} / \mathrm{m}^{3}$, tensile strength was $0.62 \mathrm{MPa}$, and elastic modulus was $0.62 \times 104 \mathrm{MPa}$. The modifications are illustrated in Figure 1 .

In comparison with the original permeameter, the improved permeameter changes the internal conductivity and rock sample specifications. The penetration path adds a guide gasket to one side of the test piece. The test piece is a cylindrical specimen with length $L$ and radius $r_{\mathrm{e}}$. A hole with radius $r_{w}$ is drilled along the axial direction, as depicted in Figure 1 .

2.2. Calculation Method for Permeability after Device Improvement. In comparison with gases, liquids have lower compressibility, higher density, and viscosity and rarely deviate from Darcy's law during permeability measurement. Under the same pressure gradient, the viscosity of the liquid volume flow is considerably higher than the gas viscosity, and a higher liquid density compensates to some extent for the influence of viscosity related to inertia resistance [33]. Thus, the vertical flow due to gravity cannot be ignored during permeability measurement. The Darcy expression of permeability measured by laminar fluid considering the effect of gravity is [34]

$$
v_{s}=\frac{q}{A}=\frac{-C_{1} k}{C_{2} \mu}\left(\frac{\mathrm{d} p}{\mathrm{~d} s}-\frac{\rho g}{C_{4}} \frac{\mathrm{d} z}{\mathrm{~d} s}\right)
$$

where $S$ is the distance along the flow direction; $\mathrm{U}_{s}$ is the volumetric flow rate of the fluid flowing through the unit void medium per unit time; $Z$ is the ordinate, increasing downward; $\rho$ is the fluid density; $(\mathrm{d} p / \mathrm{d} s)$ is the pressure gradient along the flow direction of $s ; \mu$ is the fluid viscosity; $k$ is the permeability of the medium; $q$ is the volume velocity; $A$ is the cross-sectional area perpendicular to the streamline; and $C_{1}, C_{2}$, and $C_{4}$ are constants to ensure uniformity of units.

For the transverse seepage test, there is no vertical component in the flow direction; therefore, it is considered as $(\mathrm{dz} / \mathrm{d} s)=0$. The permeability calculation formula for the fluid can be obtained by integration of Formula (1). 


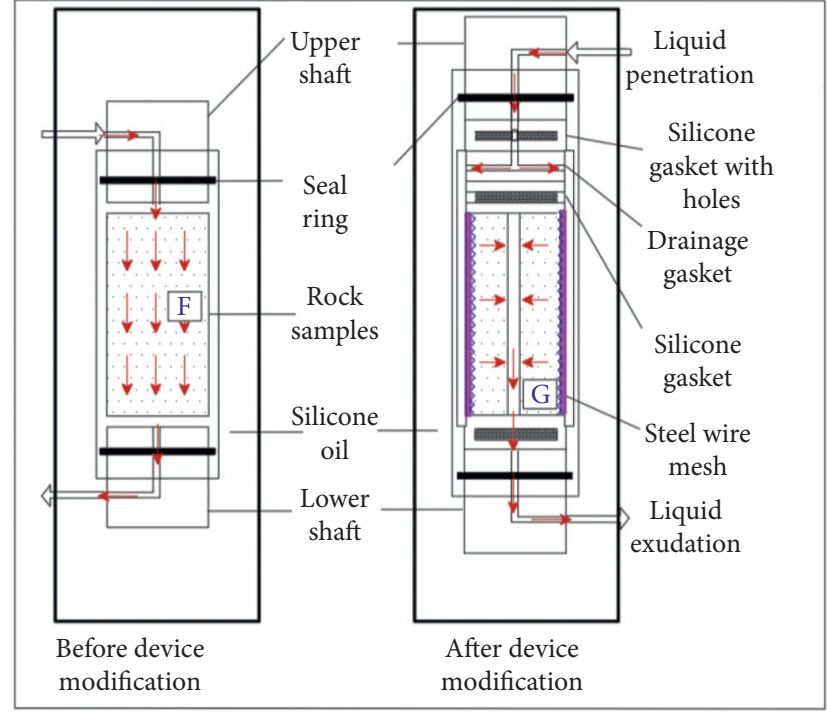

(a)

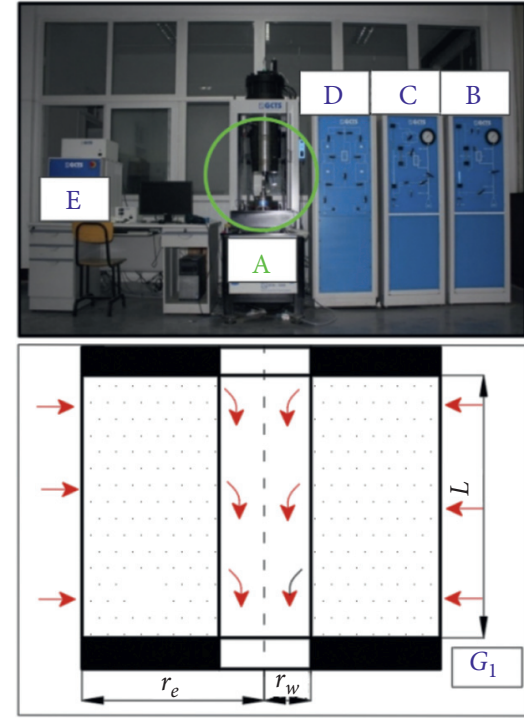

(b)

Figure 1: Comparison of flow paths before and after improvement of the RTR-1000 seepage test system. A: penetration test system; B: hole pressure supercharger; C: confining pressure supercharger; D: permeameter; E: console; F: traditional rock specimen; G: hollow cylinder rock specimen; $G_{1}$ : profile of hollow cylinder rock specimen with seal rings.

$$
k=\frac{C_{2} \mu q}{C_{1} G_{f}\left(p_{1}-p_{2}\right)},
$$

where $G_{f}$ is the geometric factor for the length, calculated by Formula (3) for radial flow.

$$
G_{f}=\frac{2 \pi L}{\ln \left[\left(r_{e} / r_{w}\right)\right]},
$$

where $L$ is the sample length; $r_{e}$ is the sample radius; and $r_{w}$ is the internal diameter.

\subsection{Experimental Process. During the test, different sensors} must be installed, including the confining pressure sensor, pore pressure sensor, axial pressure sensor, and axial displacement sensor. Because the surface of the test piece has a wire mesh, no strain gauge is directly added to the surface of the test piece; the displacement sensor on the indenter is used for calculating the strain. Using water as the permeable medium would pose a risk to the safety of the equipment owing to leakage. Thus, kerosene was used as the osmotic medium in the experiment. Before the experiment, the test piece was saturated with aviation kerosene and wrapped with a layer of steel wire mesh. The main function of the steel wire mesh is to provide a flow channel for the fluid to diffuse to the outer surface of the test piece. The hardness of the steel wire mesh should not be too high, for ease of wrapping the test piece and to avoid the risk of puncturing the heatshrinkable tube. If the diameter of the steel wire mesh hole is too large, then heat-shrinkable tube deformation may hinder the surface uniformity of the penetration medium on the surface. If the diameter of the wire mesh is too small, then the wire hole ratio may be too large, which may reduce the oil inlet cross-sectional area of the outer surface, thereby affecting the experimental results. The size of the filter mesh was between 20 and 30, the material used was 304 stainless steel, the recommended wire diameter was $0.21 \mathrm{~mm}$, and the side length of the hole was $1.10 \mathrm{~mm}$. The length of the wire mesh was slightly larger than that of the test piece; the test piece was placed between the upper head and the lower head of the pressure chamber. The expanded width of the steel wire mesh was slightly larger than the circumference of the cylindrical sample. After wrapping the steel wire mesh, a heat-shrinkable tube was placed on the periphery to seal the test piece and the upper and lower heads. In the experiment, after a specific axial confining pressure was applied, a pore pressure supercharger was used to apply the osmotic pressure $P_{1}$ using aviation kerosene, which converged horizontally from the outer surface of the sample to the center hole and flowed out of the instrument through the hole in the lower head. The experimental samples before and after the experiment are illustrated in Figure 2.

The experimental rock samples were taken from the medium coarse sandstone in the roof of the 16-3 coal seam in the Mindong mining area. The sample dimensions were as follows: height $L=100 \mathrm{~mm}$, section radius $r_{e}=25 \mathrm{~mm}$, and inner drill hole radius $r_{w}=4 \mathrm{~mm}$. The initial test confining pressure $p_{c}$ was $10 \mathrm{MPa}$, and the initial axial pressure $p_{A}$ was $10 \mathrm{MPa}$. The permeation medium was kerosene, with a dynamic viscosity $\mu=2.5 \times 10^{-3}$ Pa.s and a radial flow $G_{f}=342.86 \mathrm{~mm}$. According to the relationship between the kerosene flow rate and the time series, the flow velocity was calculated at a stable time.

\section{Results}

There are two main purposes of the experiment: to study the change rule of transverse permeability under the action of 


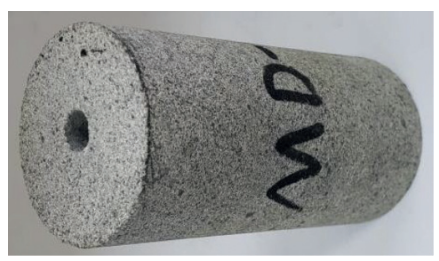

(a)

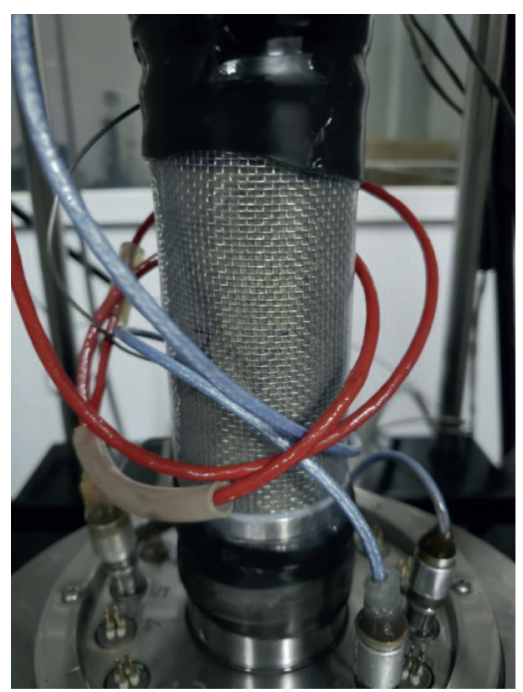

(d)

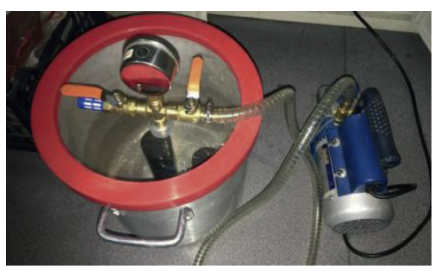

(b)

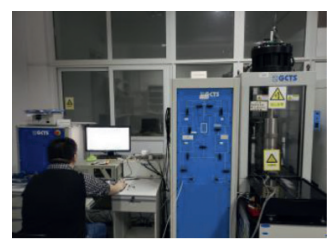

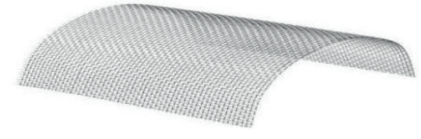

(c)

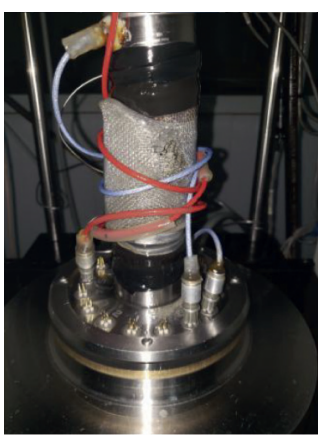

Figure 2: Diagram before and after the horizontal penetration test sample. (a) Pre-experiment (rock samples). (b) Pre-experiment (saturation device). (c) Pre-experiment (steel wire mesh). (d) Pre-experiment. (e) During experiment. (f) After completion of experiment.

mining abutment pressure in the standard environment and to provide a constitutive relation equation for the subsequent numerical construction of stress-seepage coupling and disaster prediction. According to the in situ experiments, the vertical stress and horizontal stress values of the distant rocks in the process of advancing on the working face were calculated according to the literature and the formula, and a lateral permeability test of the aquifer rocks under mininginduced stress was conducted. The test process considers the basic needs of the experiment. Each group of samples was tested three times.

To conveniently study the law of transverse permeability change and ensure the experimental effect, the unit of axial pressure increase was changed to $0.5 \mathrm{MPa}$ and $0.25 \mathrm{MPa}$, and the unit of confining pressure decrease was changed to $1 \mathrm{MPa}$. The original rock stress was $10 \mathrm{MPa}$ according to the axial pressure and the confining pressure, and the experimental process was programmed according to the loading path given in Table 1. When the flow rate was stable, the stress was changed to the next stress state in the loading path; this process continued until the rock was broken. The permeability of Darcy flow was calculated by substituting the final seepage flow results into Formula (2). The permeability coefficient and measured strain data of MD-1 sandstone are presented in Table 1 .

\section{Discussion}

In contrast to the variation rule of permeability in traditional total stress-strain, this study focuses on the variation rule of horizontal permeability of an aquifer under the influence of mining stress. The data in Table 1 consider the axial pressure as the abscissa and the permeability, confining pressure, and axial strain as the ordinate. A plot of the relationships is depicted in Figure 3.

As depicted in Figure 3, according to the stress change path of the aquifer in the process of coal seam advancement, MD-1 sandstone exhibits the correlation curve of horizontal permeability, axial pressure, and confining pressure, which are divided into compaction, multiple fluctuations, yield, and failure stages. To better explain the results, the key points in the figure are marked. Point $A$ represents the original rock stress state; point $B$ is the end point of the compaction stage; point $\mathrm{C}$ is the highest point in the middle stage; point $\mathrm{D}$ is the elastic-plastic boundary point; point $\mathrm{E}$ is the collapse failure point. 
TABLE 1: MD-1 sandstone loading path and permeability statistics.

\begin{tabular}{|c|c|c|c|c|c|c|c|c|c|}
\hline Axial compression/MPa & 10.0 & 10.5 & 11 & 11.5 & 12.0 & 12.5 & 13.0 & 13.5 & 14 \\
\hline Confining pressure, $\mathrm{MPa}$ & 9 & 9 & 9 & 9 & 9 & 9 & 9 & 9 & 9 \\
\hline Permeability, $\times 10^{-9} \mathrm{~mm}^{2}$ & 1.54 & 0.672 & 0.879 & 0.825 & 0.915 & 0.641 & 0.795 & 0.882 & 0.672 \\
\hline Axial compression, $\mathrm{MPa}$ & 14.5 & 15.0 & 15.5 & 16.0 & 16.5 & 17 & 17.5 & 18.0 & 18.5 \\
\hline Confining pressure, $\mathrm{MPa}$ & 8 & 7 & 6 & 5 & 5 & 5 & 5 & 5 & 5 \\
\hline Permeability, $\times 10^{-9} \mathrm{~mm}^{2}$ & 0.76 & 0.889 & 0.969 & 0.107 & 0.106 & 0.128 & 0.893 & 0.811 & 0.923 \\
\hline Axial compression, $\mathrm{MPa}$ & 19.0 & 19.5 & 20 & 20.5 & 21.0 & 21.5 & 22.0 & & \\
\hline Confining pressure, $\mathrm{MPa}$ & 5 & 4 & 4 & 4 & 4 & 4 & 4 & & \\
\hline Permeability, $\times 10^{-9} \mathrm{~mm}^{2}$ & 0.084 & 1.12 & 0.977 & 0.943 & 1.12 & 1 & 200 & & \\
\hline
\end{tabular}

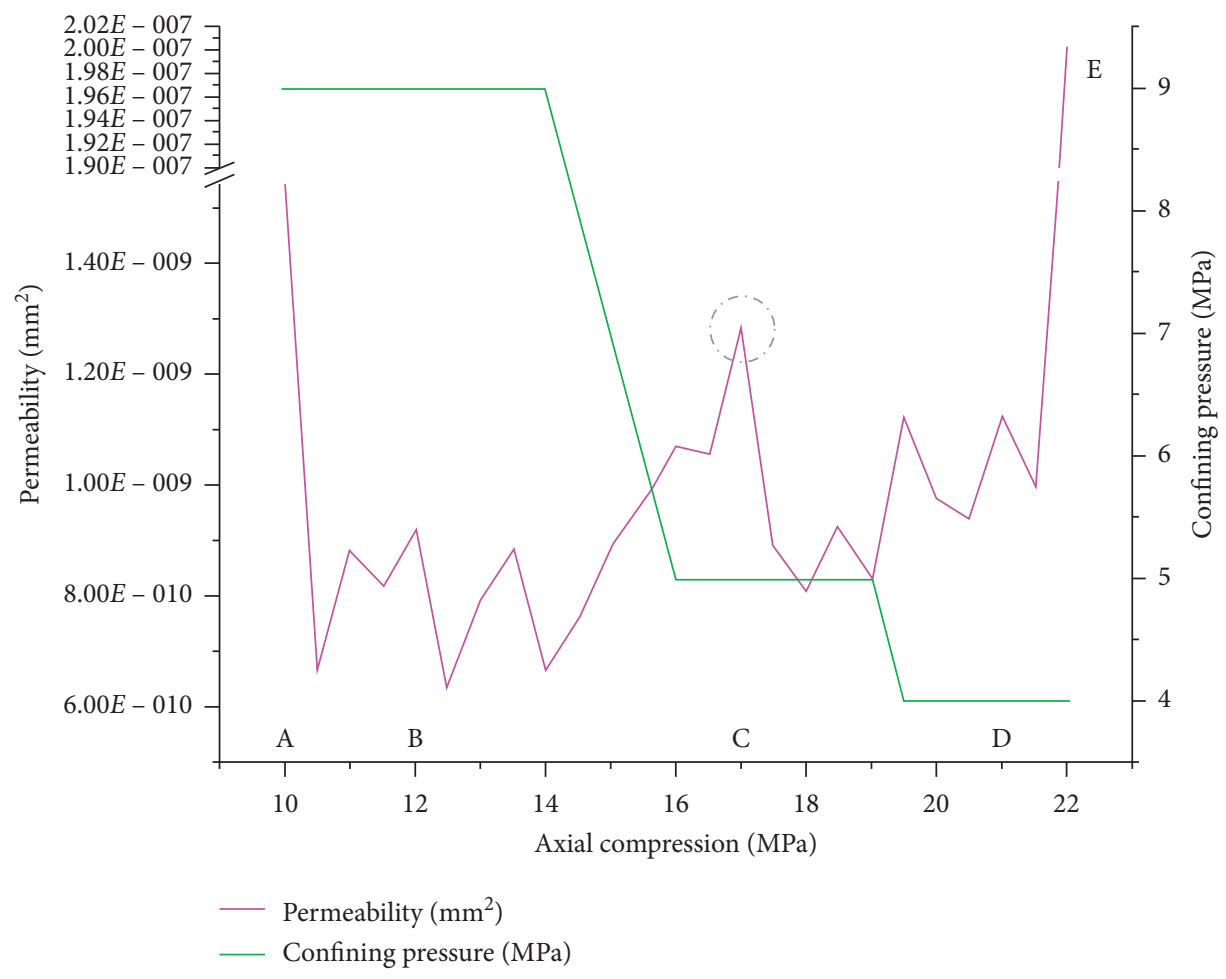

FIGURE 3: Relationship between permeability and stress state of MD-1 sandstone.

4.1. Separate Analysis of Different Sections. In AB section, when the stress increased from the original rock stress by $0.5 \mathrm{MPa}$ axial pressure, the permeability decreased sharply from $1.54 \times 10^{-9} \mathrm{~mm}^{2}$ to $6.72 \times 10 \mathrm{~mm}^{2}$, a decrease of $56 \%$, mainly due to the high porosity of the rock specimen, a water-rich aquifer rock. Although the original rock stress has been loaded, the greater porosity is compacted under the additional pressure, producing a rapid nonlinear decline trend. This stage is the first compaction period of fracture [35].

In $\mathrm{BC}$ section, in the early stage, the confining pressure remains constant at $10 \mathrm{MPa}$. As the axial pressure continues to increase by $0.5 \mathrm{MPa}$ each time, the permeability presents a small amplitude of vibration and does not show a significant increase or decrease trend. When some large fractures are compacted, the permeability of the rock decreases sharply; however, there exist a large number of parallel and vertical fractures on the cross-section of the rock in the axial direction. As the axial pressure increases, the fractures perpendicular to the axial direction are compressed, and the fractures parallel to the axial direction are opened, producing a random vibration. The later stage of the $\mathrm{BC}$ section represents the confining pressure unloading period, wherein the axial pressure is unchanged and the confining pressure is reduced. The permeability changes immediately and demonstrates an obvious increase, which is consistent with the reduction law of confining pressure [36]. Thus, the confining pressure plays a decisive role in the permeability change during this period, which includes the first shock period and the first growth period of permeability.

In the $\mathrm{CD}$ section, when the confining pressure remains at $5 \mathrm{MPa}$ and the axial pressure continues to increase at ate of $0.5 \mathrm{MPa}$ each time, the permeability coefficient exhibits a small decrease and then increases sharply, reaching $1.28 \mathrm{E}-$ $9 \mathrm{~mm}^{2}$ at the highest point in the medium term, before rapidly decreasing and fluctuating, as shown in the AC and $\mathrm{CD}$ sections. Both these sections are stress change environments in which the confining pressure first remains 


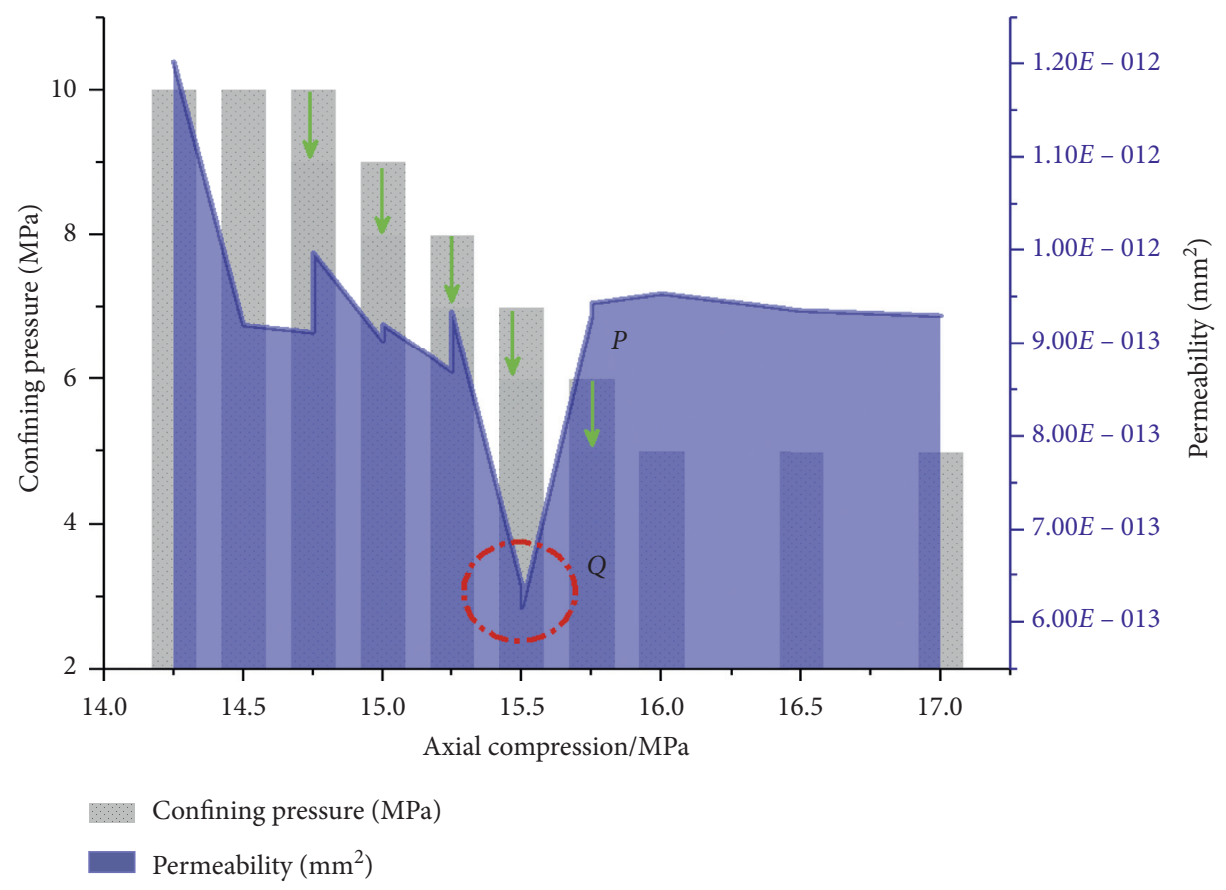

FIGURE 4: Relationship between permeability and stress state in section of nonactual changing load area in MD-2 sandstone.

unchanged and then decreases as the axial pressure gradually increases. The curve shows a high degree of similarity. The permeability first decreases rapidly and then fluctuates within a range. When the confining pressure decreases, the permeability increases immediately. After the confining pressure decreases significantly, the permeability undergoes a new equilibrium process [37]. Once the confining pressure is relieved, the compaction-fluctuation-axial pressure failure of the rock sample under axial pressure is observed. This stage includes the second compaction period, second shock period, and second growth period of permeability.

In the DE section, when the confining pressure remains at $4 \mathrm{MPa}$ and the axial pressure continues to increase at a rate of $0.5 \mathrm{MPa}$ each time, the permeability decreases twice in succession and then increases sharply to $2 \mathrm{E}-7 \mathrm{~mm}^{2}$. The final permeability is 218 times the permeability in the original rock stress state; thus, the permeability change of the aquifer rock under the mining stress loading path is completed [38].

To better understand the influence of the confining pressure and axial pressure on the permeability of the rock stratum, MD-2 is added to a section of nonactual changing load area; that is, there is no such change trend in the actual process, but it only prolongs the unloading span and has no influence on the experimental results. In the loading process, axial pressure $=14.75 \mathrm{MPa}$, confining pressure $=10 \mathrm{MPa}$, confining pressure unloads at $1 \mathrm{MPa}$, and axial pressure increases at $0.25 \mathrm{MPa}$, both of which occur separately and alternately. This stress loading path does not exist in the actual stress state of the sample. The relationship between the final permeability and the stress state is depicted in Figure 4.

The green arrows in the figure indicate that an experiment of unloading $1 \mathrm{MPa}$ under the same axial and confining pressures was conducted. The results indicate that the permeability increases four times during the five unloadings of confining pressure and the permeability decreases once. As shown by the blue line in the figure, the horizontal permeability decreases slightly in the $\mathrm{Q}$ area. After five times of increasing $0.25 \mathrm{MPa}$ under the same axial and confining pressures, the permeability decreases four times, as shown by the magenta line in the figure. A significant increase in lateral permeability occurs in the QP section, demonstrating that the increase in axial pressure tends to reduce the lateral permeability, mainly due to the effect of axial compression and compaction. The decrease in confining pressure tends to increase the lateral permeability, mainly due to the effect of confining pressure unloading and expansion. However, when the two factors act together, the differences in the action weight result in a wave of permeability. The influence weight of the confining pressure and axial pressure on the transverse permeability is related to the stress environment and the damage state of the rock sample. The crack direction and propagation scale of the internal fracture of the rock affect the transverse permeability within a small range.

The two sets of data reflect consistency at the macro level. The permeability coefficient exhibits a sharp decline and then stabilizes and fluctuates slightly, surging with the change in stress state. This process differs from the rule shown in the traditional sandstone total stress-strain relationship curve. First, the sandstone permeability exhibits a rapid downward trend in the small range of stress change at the initial stage of loading. Subsequently, the permeability is low with small fluctuations. Second, it exhibits a small wave peak value in the middle-later period of loading; however, the wave peak value is lower than the permeability under the original rock stress. Third, the rock undergoes a short plastic stage and a failure stage. The main reason why these three points differ from the traditional sandstone total stressstrain curve is that the coal seam roof strata simulated in this 
experiment are under the influence of mining stress. The relationship between stress and permeability, different loading paths, and the experimental research subject is based on the lateral permeability of the rock and the different seepage directions. For the law of the experimental results, the pressure loading path was drawn according to the change trend of the confining pressure of No. III. In numerical simulation research, only the vertical pressure and permeability must be combined. The relationship between the horizontal permeability and the axial pressure of sandstone obtained from the experiment is divided into two sections; each section is fitted with a quadratic curve equation, which is used as the constitutive relation equation for later numerical simulation of the correlation between stress and permeability. This formula is not a theoretical derivation but has significance in practical engineering, particularly for mining areas under complex hydrogeological conditions. A more accurate constitutive equation has a better effect on the prediction of water inflow and the migration law of the groundwater flow field.

\section{Conclusion}

(1) Through the design of a hollow cylinder specimen, the original permeability testing experiment was changed from a vertical seepage mode of the permeability medium to a seepage mode from the outside to the inside. Furthermore, through the derivation transformation of the Darcy expression of the permeability measurement of the laminar fluid considering the influence of gravity, an accurate improved permeability calculation formula that is suitable for similar experimental conditions can be deduced. The measurement of horizontal permeability is a scientific reference.

(2) Through the improved test method, it was found that the sandstone specimen increased the axial pressure and decreased the confining pressure on the basis of the original rock stress. With the change in stress, the permeability first decreased sharply, then stabilized, fluctuated slightly, and increased sharply.

\section{Data Availability}

The datasets generated for this study are available from the corresponding author upon request.

\section{Conflicts of Interest}

The authors declare that they have no conflicts of interest.

\section{Acknowledgments}

This work was financially supported by the National Natural Science Foundation of China (no. 51774009), Anhui Provincial Department of Education (no. KJ2019A0134), Young Teacher Scientific Research Fund Project of Anhui University of Science and Technology (no. QN2018123), and Rheological Characteristics of Rock Damage in Deep Mining
Areas and Their Effect on Roadway Stability (SKLMRDPC19ZZ08).

\section{References}

[1] X. Qian, C. Xia, Y. Gui, X. Zhuang, and Q. Yu, "Study on flow regimes and seepage models through open rough-walled rock joints under high hydraulic gradient," Hydrogeology Journal, vol. 27, no. 4, pp. 1329-1343, 2019.

[2] Z.-Q. Yin, Z. X. Hu, Z. D. Wei et al., "Assessment of blastinginduced ground vibration in an open-pit mine under different rock properties," Advances in Civil Engineering, vol. 2018, Article ID 4603687, 10 pages, 2018.

[3] Z. Yin, W. Chen, H. Hao et al., "Dynamic compressive test of gas-containing coal using a modified split Hopkinson pressure bar system," Rock Mechanics and Rock Engineering, vol. 53, no. 2, pp. 815-829, 2020.

[4] Z. Wen, S. Jing, Y. Jiang et al., "of the fracture law of overlying strata under water based on the flow-stress-damage model," Geofluids, vol. 201912 pages, 2019.

[5] F. Tian, J. Yan, X. Li, and S. Luo, "A peak-strength strain energy storage index for rock burst proneness of rock materials," International Journal of Rock Mechanics and Mining Sciences, vol. 117, pp. 76-89, 2019.

[6] H. Zhang, M. Tu, H. Cheng, and Y. Tang, "Breaking mechanism and control technology of sandstone straight roof in thin bedrock stope," International Journal of Mining Science and Technology, vol. 30, no. 2, pp. 259-263, 2020.

[7] D. T. Snow, "Rock fracture spacings, openings and porosities," Journal of the Soil Mechanics \& Foundations Division, vol. 94, no. 1, pp. 73-91, 1968.

[8] M. Oda, "An equivalent continuum model for coupled stress and fluid flow analysis in jointed rock masses," Water Resources Research, vol. 22, no. 13, pp. 1845-1856, 1986.

[9] M. Oda, "A method for evaluating the effect of crack geometry on the mechanical behavior of cracked rock masses," $M e$ chanics of Materials, vol. 2, no. 2, pp. 163-171, 1983.

[10] M. Oda, Y. Hatsuyama, and Y. Ohnishi, "Numerical experiments on permeability tensor and its application to jointed granite at Stripa Mine, Sweden," Journal of Geophysical Research Solid Earth, vol. 92, no. 8, 1987.

[11] N. Barton, K. S. Bandis, and K. Bakhtar, "Strength, deformation and conductivity coupling of rock joints," International Journal of Rock Mechanics and Mining Sciences \& Geomechanics Abstracts, vol. 22, no. 3, pp. 121-140, 1985.

[12] V. Reichenberger, H. Jakobs, P. Bastian, and R. Helmig, "A mixed-dimensional finite volume method for two-phase flow in fractured porous media," Advances in Water Resources, vol. 29, no. 7, pp. 1020-1036, 2006.

[13] H. Hoteit and A. Firoozabadi, "An efficient numerical model for incompressible two-phase flow in fractured media," $A d$ vances in Water Resources, vol. 31, no. 6, pp. 891-905, 2008.

[14] R. Helmig, B. Flemisch, M. Wolff, A. Ebigbo, and H. Class, "Model coupling for multiphase flow in porous media," Advances in Water Resources, vol. 51, pp. 52-66, 2013.

[15] A. Peratta and V. Popov, "A new scheme for numerical modelling of flow and transport processes in 3D fractured porous media," Advances in Water Resources, vol. 29, no. 1, pp. 42-61, 2006.

[16] J. Zhao, L. Yin, and W. Guo, "Stress-seepage coupling of cataclastic rock masses based on digital image technologies," Rock Mechanics and Rock Engineering, vol. 51, no. 8, pp. 2355-2372, 2018.

[17] C. Wang and Q.-Y. Zhang, "Study of the crack propagation model under seepage-stress coupling based on XFEM," 
Geotechnical and Geological Engineering, vol. 35, no. 5, pp. 2433-2444, 2017.

[18] H. Gui, H. Qiu, W. Qiu, S. Tong, and H. Zhang, "Overview of goaf water hazards control in China coalmines," Arabian Journal of Geosciences, vol. 11, no. 3, p. 49, 2018.

[19] J. Yi, H. L. Xing, J. J. Wang, and Z. H. Xia, Y. Jing, "Pore-scale study of the effects of surface roughness on relative permeability of rock fractures using lattice Boltzmann method," Chemical Engineering Science, p. 209, Article ID 115178, 2019.

[20] W. J. Xiao, D. M. . Zhang, and X. J. Wang, "Experimental study on progressive failure process and permeability characteristics of red sandstone under seepage pressure," Engineering Geology, vol. 265, Article ID 105406, 2020.

[21] Q. G. Tan, J. Y. Li, Y. L. . Kang, X. Zhang et al., "Changes in pore structures and porosity-permeability evolution of salinelacustrine carbonate reservoir triggered by fresh water-rock reaction," Journal of Hydrology, vol. 580, Article ID 124375 , 2019.

[22] F. M. R. Ferfera, J. P. Sarda, M. Boutéca, and O. Vincké, "Experimental study of monophasic permeability changes under various stress paths," International Journal of Rock Mechanics and Mining Sciences, vol. 34, no. 3-4, 370 pages, Article ID 2147483647, 1997.

[23] X. Chen, J. Yu, C. a. Tang, H. Li, and S. Wang, "Experimental and numerical investigation of permeability evolution with damage of sandstone under triaxial compression," Rock Mechanics and Rock Engineering, vol. 50, no. 6, pp. 1529-1549, 2017.

[24] S.-Q. Yang, P. Xu, and Y.-H. Huang, "Experimental investigation on triaxial mechanical and permeability behavior of sandstone after exposure to different high temperature treatments," Geothermics, vol. 69, pp. 93-109, 2017.

[25] P. F. Shan and X. P. Lai, "Numerical simulation of the fluidsolid coupling process during the failure of a fractured coalrock mass based on the regional geostress," Transport in Porous Media, vol. 124, no. 3, pp. 1061-1079, 2018.

[26] B. Sheikh and A. Pak, "Numerical investigation of the effects of porosity and tortuosity on soil permeability using coupled three-dimensional discrete-element method and lattice Boltzmann method," Physical Review E, vol. 91, no. 5, Article ID 053301, 2015.

[27] W. Guo, J. Zhao, and L. Yin, "Simulating research on pressure distribution of floor pore water based on fluid-solid coupling," Arabian Journal of Geosciences, vol. 10, p. 5, 2017.

[28] J. Zhang, F. J. Biao, S. C. Zhang, and X. X. Wang, "A numerical study on interference between different layers for a layer-bylayer hydraulic fracture procedure," Petroleum Science and Technology, vol. 32, no. 12, pp. 1512-1519, 2014.

[29] W. T. Ding and W. J. Xu, "Study on the multiphase fluid-solid interaction in granular materials based on an LBM-DEM coupled method," Powder Technology, vol. 335, pp. 301-314, Article ID S0032591018303590, 2018.

[30] J. Q. Shi and S. Durucan, "Modelling laboratory horizontal stress and coal permeability data using S\&D permeability model," International Journal of Coal Geology, vol. 131, pp. 172-176, 2014.

[31] E. P. Weeks, "Determining the ratio of horizontal to vertical permeability by aquifer-test analysis," Water Resources Research, vol. 5, no. 1, pp. 196-214, 1969.

[32] A. Wang and V. S. Yakushev, "Analytical model of the linear inflow to a horizontal well with hydraulic fractures in lowpermeability reservoirs," Fluid Dynamics, vol. 52, no. 3, pp. 351-362, 2017.
[33] D. L. Brown, Multiscale methods for fluid-structure interaction with applications to deformable porous media, Ph.D. thesis, Dissertations \& Theses-Gradworks, Cincinnati, USA, 2013.

[34] S. A. Mathias, S. Nielsen, and R. L. Ward, "Storage coefficients and permeability functions for coal-bed methane production under uniaxial strain conditions," Transport in Porous Media, vol. 130, no. 2, pp. 627-636, 2019.

[35] A. A. DiGiovanni, D. J. Fredrich, W. A. Holcomb, and W. Olsson, "Microscale damage evolution in compacting sandstone," Geological Society, London, Special Publications, vol. 289, no. 1, pp. 89-103, 2007.

[36] Y. L. Zhao, J. Z. Tang, Y. Chen et al., "Hydromechanical coupling tests for mechanical and permeability characteristics of fractured limestone in complete stress-strain process," Environmental Earth Sciences, vol. 76, no. 1, pp. 1-18, 2017.

[37] Y. Zhao, L. Zhang, W. Wang, C. Pu, W. Wan, and J. Tang, "Cracking and stress-strain behavior of rock-like material containing two flaws under uniaxial compression," Rock Mechanics and Rock Engineering, vol. 49, no. 7, pp. 26652687, 2016.

[38] L. Wang, J. F. Liu, J. L. Pei, H. N. Xu, and Y. Bian, "Mechanical and permeability characteristics of rock under hydro-mechanical coupling conditions," Environmental Earth Sciences, vol. 73, no. 10, pp. 5987-5996, 2015. 